\section{AB0980 FACTORS ASSOCIATED WITH LOSS OF CARTILAGE IN} KNEE OSTEOARTHRITIS

V. Vardanyan ${ }^{1}$, V. Mukuchyan ${ }^{2}$, A. Kirakosyan' ${ }^{2} .{ }^{1}$ Internal Medicine, Yerevan State Medical University; ${ }^{2}$ Rheumatology, "Erebouni" Medical Center, Yerevan, Armenia

Background: Osteoarthritis $(O A)$ is one of the leading causes of pain and disability worldwide. The structural changes in knee OA are characterised mainly by the progressive loss of cartilage, which is associated with additional structural changes such as subchondral bone lesions and alterations in the menisci. Unfortunately, these changes cannot be effectively treated conservatively. Further determination of modifiable risk factors of cartilage loss is extremely important for improvement of OA management.

Objectives: The main objective of the study was the determination of factors associated with loss of cartilage in knee OA.

Methods: 294 patients ( 277 female, 17 male, mean age 55, 09 $\pm 8,56$ years) with newly diagnosed mild to moderate primary knee osteoarthritis were investigated. $X$-ray and ultrasound examinations of knee joints were performed. The thickness of cartilage and synovial layer, as well as presence of synovitis, tendinitis, osteophytes, Baker's cysts, tear of meniscus were determined. For determination of factors associated with loss of cartilage both univariate and multivariate analyses were performed. The data is introduced as odds ratios (OR) with $95 \%$ confidence interval $(\mathrm{Cl})$. The results were considered significant when $\mathrm{p}<0.05$

Results: Expressed thinning of cartilage ( $\leq 2 \mathrm{~mm}$ ) was observed in $216(73,5 \%)$ patients, abnormal thickening of synovial layer ( $\geq 3 \mathrm{~mm})$ - in $76(25,9 \%)$, synovitis - in $88(29,9 \%)$, tendinitis - in $38(12,9 \%)$, osteophytes - in $273(92,9 \%)$, Baker's cysts -in $93(31,6 \%)$, tears of meniscus - in $93(31,6 \%)$ patients.

Univariate analysis had shown that loss of cartilage $(\leq 2 \mathrm{~mm})$ was significantly $(\mathrm{p}<0,05)$ associated with age $>55(\mathrm{OR} / 95 \% \mathrm{Cl} /=2,0 / 1,3-3,4 /, \mathrm{p}<0,05)$, thickening of synovial layer (OR/95\% Cl/=2,9/1,5-6,2/, $\mathrm{p}<0,01)$, synovitis $(\mathrm{OR} / 95 \% \mathrm{Cl} /=2,6$ / $1,3-5,0 /, \mathrm{p}<0,01)$ and osteophytes $(\mathrm{OR} / 95 \% \mathrm{Cl} /=14,8 / 4,8-45,5 /, \mathrm{p}<0,01)$. Following factors were then stepwise included in the model of multivariate logistic regression: age $>55$, thickening of synovial layer and osteophytosis. According to results of analysis, age $>55$ had lost its significance, was evaluated as confounding factor $(\mathrm{OR} / 95 \% \mathrm{Cl} /=1,2 / 0,7-2,2 /, \mathrm{p}=0,4)$ and excluded from the model. Finally, loss of cartilage (thickness $\leq 2 \mathrm{~mm}$ ) was found to be significantly and independently associated with abnormal thickening of synovial layer $(\mathrm{OR} / 95 \% \mathrm{Cl} /=2,8 / 1,3-6,0$, $\mathrm{p}<0,05)$ and osteophytosis (OR/95\% Cl/ $=12,8 / 3,9-41,6 /, \mathrm{p}<0,01)$.

Conclusions: A positive association of cartilage loss with abnormal thickening of synovial layer (with further development of synovitis) and presence of osteophytes was determined. While cartilage loss and meniscal damage are not yet clearly treatable, treatments targeting inflammation within the joint are available. Thereby, forehanded treatment of secondary inflammatory conditions of joint, as well as strategies, directed toward dissection of osteophytes, can decrease cartilage loss and structural damage in OA.

\section{REFERENCES:}

[1] Martel-Pelletier J, Lajeunesse D, Pelletier JP: Etiopathogenesis of osteoarthritis. Arthritis and Allied Conditions: A Textbook of Rheumatology. Edited by: Koopman WJ, Moreland LW. 2005, Baltimore, MD: Lippincott, Williams \& Wilkins, 2199-2226.

Disclosure of Interest: None declared DOI: 10.1136/annrheumdis-2018-eular.3761

\section{AB0981 $\quad$ PRIORITIES FOR OSTEOARTHRITIS RESEARCH SHOULD BE DONE IN CHINA}

Y. Mei ${ }^{1}$, S. Guan ${ }^{1}$, Y. Shi ${ }^{1}$, D.J. Hunter ${ }^{2}$, Z. Zhang ${ }^{1} .{ }^{1}$ the First Affiliated Hospital of Harbin Medical University, Harbin, China; ${ }^{2}$ University of Sydney, Sydney, Australia

Background: Osteoarthritis $(O A)$ exacts a tremendous toll on patients and society due to limited treatment options and unclear pathological mechanism. Furthermore, rising rates of obese and senior population are predicted to increase the prevalence of $\mathrm{OA}$ in the coming years in China. With the development of economy, Chinese government increasingly encourage researches for prevention and treatment of chronic diseases including OA. However, the studies of OA in China remain inadequate.

Objectives: This study was designed, in collaboration with Chinese rheumatologists, to investigate key priorities for $O A$ in the next five years to make use of limited funding and resource in China. In addition, it will also help researchers, especially Chinese rheumatologists, to get more support from the government, which will push OA studies faster towards elusive breakthroughs. Therefore, it will improve prognosis and health and quality of life for OA patients.

Methods: The study comprised 4 stages, including 1) Identify research priorities for the next five years that match the strengths and expertise of Chinese OA researchers at the OA potential priority-setting summit. 2) Initial ranking of suggested research priorities using 1000Minds software prior to the summit. 3) Dis cussion and selection of top five thematic priorities from the original list of 10 priorities at the final OA priority-setting summit. 4) Final ranking of five research priorities by all attendees at the summit and design experimental aims and approaches for the priorities. Finally, the top three priorities from five prioritised topics were selected.

Results: 39 Chinese rheumatologists attended stage one and identified 10 priorities in the next five years. There were 313 Chinese rheumatologists invited to participate in stage two, among them, 104 rheumatologists finished the process and identified the initial prioritised rankings of the research topics. 29 Chinese rheumatologists attended the final OA priority-setting summit. Through these multistage processes, three key topics about OA were identified as essential and were prioritised. These three priorities include targeting inflammation should be a focus of research in osteoarthritis, seeking biomarkers for osteoarthritis diagnosis and monitoring, research into early OA would be expected to understand OA across disease development and translate to better therapy. Experimental aims included to detect inflammatory in OA pathology, to identify well-established criteria for early $\mathrm{OA}$, to protect and repair chondrocytes and cartilage, and the approaches included longitudinal study, placebo-controlled randomised controlled trial, anima experiments.

Conclusions: Chinese rheumatologists identified OA priorities for the first time, and these key topics would have guiding effect significantly on Chinese OA research in the future.

\section{REFERENCES}

[1] Duarte PEREIRA, Elisabete RAMOS, Jaime BRANCO, Osteoarthritis Acta Med Port 2015 Jan-Feb;28(1):99-106

[2] Harris $\mathrm{H}$, Crawford $\mathrm{A}$. Recognizing and managing osteoarthritis. Nursing. 2015 Jan;45(1):36-42; quiz 42-3. doi:10.1097/01. NURSE.0000458918.87973.15

[3] Sinusas K. Osteoarthritis: diagnosis and treatment. Am Fam Physician. 2012 Jan 1;85(1):49-56

Disclosure of Interest: None declared

DOI: 10.1136/annrheumdis-2018-eular.6606

\section{AB0982 PREVALENCE OF OSTEOARTHRITIS IN HIGH ALTITUDE AREA OF TIBET}

Y. Liang, Y. Mei, S. Guan, W. Sun, W. Wang, F. Teng, X. Han, Z. Zhang. the First Affiliated Hospital of Harbin Medical University, Harbin, China

Background: Osteoarthritis is a degenerative joint disease; the specific etiology and pathogenesis are unclear. The prevalence of osteoarthritis of subtypes of joints is different, whereas the most common osteoarthritis occurs in knees. In China, the prevalence of osteoarthritis is about $15 \%$, the prevalence of the patients over 40 years old is $10 \% \sim 17 \%$, and $50 \%$ over the age of 60 , while over 75 years old was as high as $80 \%$. Few data exist regarding the prevalence of OA in plateau in China.

Objectives: To investigate the prevalence of osteoarthritis $(O A)$ in Jiuhe village which is located about 3.5 kilometres high of Qonggyai County of Tibet and the associated factors in order to provide the guidance for the prevention and treatment of $O A$

Methods: The participants in this analysis were all the resident people aged 50 years and above in Jiuhe village, Qonggyai County of Tibet. All subjects were invited to completed an questionnaire, physical examination and radiographic examination. The questionnaire included sex, age, body mass index(BMI) and dietary habit.

Results: A total of 136 participants aged $50-86$ years were enrolled, including 47 males with mean age of $62.00 \pm 9.11$ years, and 89 females with mean age of $61.30 \pm 9.52$ years. Altogether 102 knee OA can be diagnosed, the total prevalence of knee OA was $75.00 \%$. Knee OA occurred in $78.72 \%$ of male and $73.03 \%$ of female. The prevalence of knee OA combined with hand OA was $19.12 \%$, and $21.28 \%$ for male and $17.98 \%$ for female. The prevalence of knee OA combined with hand $O A$ increased with age in the female, no such trend was observed in male or in only knee OA patients. No significant difference was found about sex BMI, drinking between the OA patients and the controls.

Conclusions: The prevalence of osteoarthritis in Jiuhe village, plateau of Tibet was significantly high. The prevalence in male is higher than that in female. The prevalence of knee OA combined with hand OA increased with age in the female.

Acknowledgements: Create China Hearts Foundation

Disclosure of Interest: None declared

DOI: 10.1136/annrheumdis-2018-eular.6679 